\title{
Symmetry Plus Quasi Uniform Association Model and Its Orthogonal Decomposition for Square Contingency Tables
}

\author{
Kouji Yamamoto \\ Osaka University Hospital, Suita City, Japan, yamamoto-k@hp-crc.med.osakau.ac.jp \\ Sadao Tomizawa \\ Tokyo University of Science, Noda City, Japan, tomizawa@is.noda.tus.ac.jp
}

Follow this and additional works at: http://digitalcommons.wayne.edu/jmasm

Part of the Applied Statistics Commons, Social and Behavioral Sciences Commons, and the Statistical Theory Commons

\footnotetext{
Recommended Citation

Yamamoto, Kouji and Tomizawa, Sadao (2010) "Symmetry Plus Quasi Uniform Association Model and Its Orthogonal Decomposition for Square Contingency Tables," Journal of Modern Applied Statistical Methods: Vol. 9 : Iss. 1 , Article 25. DOI: $10.22237 /$ jmasm/1272687840

Available at: http://digitalcommons.wayne.edu/jmasm/vol9/iss1/25
}

This Regular Article is brought to you for free and open access by the Open Access Journals at DigitalCommons@WayneState. It has been accepted for inclusion in Journal of Modern Applied Statistical Methods by an authorized editor of DigitalCommons@WayneState. 


\section{Symmetry Plus Quasi Uniform Association Model and Its Orthogonal Decomposition for Square Contingency Tables}

\author{
Kouji Yamamoto \\ Osaka University Hospital, \\ Suita City, Japan
}

\author{
Sadao Tomizawa \\ Tokyo University of Science, \\ Noda City, Japan
}

A model is proposed having the structure of both symmetry and quasi-uniform association (SQU model) and provides a decomposition of the SQU model. It is also shown with examples that the test statistic for goodness-of-fit of the SQU model is asymptotically equivalent to the sum of those for the decomposed models.

Key words: Likelihood ratio statistic, marginal homogeneity, quasi-symmetry, quasi-uniform association, separability.

\section{Introduction}

For the $r \times r$ square contingency table, let $p_{i j}$ denote the probability that an observation will fall in the $i^{\text {th }}$ row and $j^{\text {th }}$ column of the table ( $i=1, \ldots, r ; j=1, \ldots, r)$. For the analysis of twoway contingency tables with ordered categories, Goodman (1979) considered some association models, for example, the uniform association model, which is a generalization of the independence model. Goodman (1979) also observed that regular multiplicative models for ordinal variables fit square contingency tables well when the cells on the main diagonal are ignored, thus, he proposed the quasi-uniform association (QU) model, defined by

$$
p_{i j}=\left\{\begin{array}{cc}
\mu \alpha_{i} \beta_{j} \theta^{i j} & (i \neq j), \\
\psi_{i i} & (i=j) .
\end{array}\right.
$$

when $\theta=1$, this model is the quasi-

Kouji Yamamoto is an Assistant Professor on Center for Clinical Investigation and Research. Email him at: yamamoto-k@hp-crc.med.osakau.ac.jp. Sadao Tomizawa is a Professor on the Faculty of Science and Technology in the Department of Information Sciences. Email him at: tomizawa@is.noda.tus.ac.jp. independence (QI) model (Bishop, Fienberg \& Holland, 1975, p. 178).

The symmetry (S) model considered by Bowker (1948) is defined by

$$
p_{i j}=\psi_{i j} \quad(i=1, \ldots, r ; j=1, \ldots, r),
$$

where $\psi_{i j}=\psi_{j i}$ (Bishop, et al., 1975, p. 282). This model describes a structure of symmetry of the cell probabilities $\left\{p_{i j}\right\}$ with respect to the main diagonal of the table. The quasi-symmetry (QS) model considered by Caussinus (1965) is defined by

$$
p_{i j}=\mu \alpha_{i} \beta_{j} \psi_{i j} \quad(i=1, \ldots, r ; j=1, \ldots, r),
$$

where $\psi_{i j}=\psi_{j i}$. The odds ratio for rows $i$ and $j(>i)$, and columns $s$ and $t(>s)$ are denoted by $\theta_{(i j ; s t)}$; thus,

$$
\theta_{(i j ; s t)}=\frac{\left(p_{i s} p_{j t}\right)}{\left(p_{j s} p_{i t}\right)}
$$

Using odds ratios, the QS model may be expressed as

$$
\theta_{(i j ; s t)}=\theta_{(s t ; i j)} \quad(i<j ; s<t) .
$$




\section{ORTHOGONAL DECOMPOSITION FOR QUASI UNIFORM ASSOCIATION MODEL}

Therefore this model indicates a structure of symmetry with respect to the odds ratios. A special case of this model obtained by putting $\left\{\alpha_{i}=\beta_{i}\right\}$ is the $\mathrm{S}$ model. Also, each of the QI and QU models is a special case of the QS model. The marginal homogeneity $(\mathrm{MH})$ model is defined by

$$
p_{i \cdot}=p_{\cdot i} \quad(i=1, \ldots, r)
$$

where $\quad p_{i \cdot}=\sum_{t=1}^{r} p_{i t} \quad$ and $\quad p_{\cdot i}=\sum_{s=1}^{r} p_{s i}$ (Stuart, 1955).

Decomposition of the $\mathrm{S}$ Model (Caussinus, 1965)

Theorem 1

The S model holds if and only if both the QS and MH models hold.

The symmetry plus quasi-independence (SQI) model introduced by Goodman (1985) is defined by

$$
p_{i j}=\left\{\begin{array}{cc}
\mu \alpha_{i} \alpha_{j} & (i \neq j), \\
\psi_{i i} & (i=j) .
\end{array}\right.
$$

This model is a special case of the $\mathrm{S}$ model obtained by substituting $\left\{\psi_{i j}=\alpha_{i} \alpha_{j}\right\}$ for $i \neq j$.

The purpose of this study is to: (1) propose a model that can be used to simultaneously study both symmetry and quasiuniform association (the SQU model), (2) provide decomposition of the proposed model, and (3) show the orthogonality of decomposition with respect to the goodness-of-fit test statistic.

Figure 1. Relationships among the Models

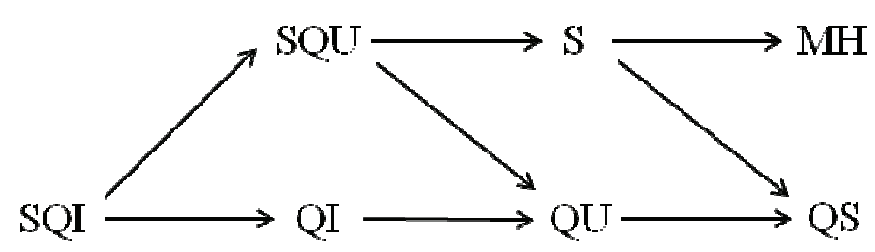

Proposed Model

Consider a model defined by:

$$
p_{i j}=\left\{\begin{array}{cc}
\mu \alpha_{i} \alpha_{j} \theta^{i j} & (i \neq j), \\
\psi_{i i} & (i=j) .
\end{array}\right.
$$

This model indicates that both the $\mathrm{S}$ and QU models hold simultaneously. Thus, this model shall be referred to as the symmetry plus quasiuniform association (SQU) model. The SQU model is an extension of the SQI model. Under the SQU model, the row marginal distribution is identical with the column marginal distribution. Using odds ratios, the SQU model may be expressed as

$$
\theta_{(i j ; s t)}=\theta^{(j-i)(t-s)} \quad(i \neq s, i \neq t, j \neq s, j \neq t) .
$$

This model has uniform local association for cells off the main diagonal of the table. Figure 1 shows the relationships among the models.

Decompositions of the Models

Theorem 2

The SQU model holds if and only if both the QU and MH models hold.

Theorem 2 Proof

If the SQU model holds, then the QU and $\mathrm{MH}$ models hold. Conversely, if both the QU and MH models hold, then the QS model holds. Therefore, from Theorem 1 it may be stated that the $\mathrm{S}$ model holds. Thus, SQU model holds. The proof is completed and the following corollary is obtained because the SQI model is a special case of the SQU model with $\theta=1$.

\section{Corollary 1}

The SQI model holds if and only if both the QI and MH models hold.

Orthogonality of Decomposition of Test Statistic for the Models

Let $n_{i j}$ denote the observed frequency in the $(i, j)^{\text {th }}$ cell of the table $(i=1, \ldots, r ; j=1, \ldots, r)$. Assume that a multinomial distribution applies to the $r \times r$ table. The maximum likelihood estimates of 
expected frequencies under the models described in this paper could be obtained using an iterative procedure, for example, the general iterative procedure for log-linear models of Darroch and Ratcliff (1972) or using the Newton-Raphson method to the log-likelihood equations.

Each model can be tested for goodnessof-fit by, for example, the likelihood ratio Chisquared statistic with the corresponding degrees of freedom (df). The numbers of $\mathrm{df}$ for the SQU, QU, and MH models are $r^{2}-2 r-1, r(r-3)$, and $r-1$, respectively. Let $G^{2}(\Omega)$ denote the likelihood ratio statistic for testing the goodnessof-fit of model $\Omega$. Thus

$$
G^{2}(\Omega)=2 \sum_{i=1}^{r} \sum_{j=1}^{r} n_{i j} \log \left(\frac{n_{i j}}{\hat{m}_{i j}}\right),
$$

where $\hat{m}_{i j}$ is the maximum likelihood estimate of expected frequency $m_{i j}$ under model $\Omega$.

For the analysis of contingency tables, Lang and Agresti (1994) and Lang (1996) considered the simultaneous modeling of the joint distribution and of the marginal distribution. Aitchison (1962) discussed the asymptotic separability, which is equivalent to the orthogonality in Read (1977) and the independence in Darroch and Silvey (1963) of the test statistic for goodness-of-fit of two models (also see Lang \& Agresti, 1994; Lang, 1996; Tomizawa \& Tahata, 2007; Tahata \& Tomizawa, 2008).

Theorem 3 holds:

The following asymptotic equivalence

$$
G^{2}(S Q U) \simeq G^{2}(Q U)+G^{2}(M H) .
$$

The number of $\mathrm{df}$ for the SQU model equals the sum of the numbers of $\mathrm{df}$ for the $\mathrm{QU}$ and $\mathrm{MH}$ models.

Theorem 3 Proof

The QU model may be expressed in a log-linear form

$$
\begin{aligned}
& \log p_{i j}=\mu^{*}+\alpha_{i}^{*}+\beta_{j}^{*}+i j \theta^{*}+\psi_{i i}^{*} I(i=j) \\
& (i=1, \ldots, r ; j=1, \ldots, r),
\end{aligned}
$$

where $\mu^{*}=\log \mu, \alpha_{i}^{*}=\log \alpha_{i}$ (and so on) with $\alpha_{r}^{*}=\beta_{r}^{*}=0$ without loss of generality, and where $I(i=j)=1$ if $i=j$ and 0 otherwise. Let

$$
p=\left(p_{11}, \ldots, p_{1 r}, p_{21}, \ldots, p_{2 r}, \ldots, p_{r r}\right)^{\prime},
$$

and

$$
\beta=\left(\mu^{*}, \beta_{1}, \beta_{2}, \beta_{12}\right)^{\prime}
$$

where "'" denotes the transposed,

$$
\beta_{1}=\left(\alpha_{1}^{*}, \ldots, \alpha_{r-1}^{*}\right), \beta_{2}=\left(\beta_{1}^{*}, \ldots, \beta_{r-1}^{*}\right),
$$

and

$$
\beta_{12}=\left(\theta^{*}, \psi_{11}^{*}, \ldots, \psi_{r r}^{*}\right) .
$$

The QU model is then expressed as

$$
\log p=X \beta=\left(1_{r^{2}}, X_{1}, X_{2}, X_{12}\right) \beta,
$$

where $X$ is the $r^{2} \times 3 r$ matrix and $1_{s}$ is the $s \times 1$ vector of 1 elements,

$$
X_{1}=\left[\begin{array}{c}
I_{r-1} \otimes 1_{r} \\
O_{r, r-1}
\end{array}\right] ; \text { the } r^{2} \times(r-1) \text { matrix, }
$$

$$
X_{2}=1_{r} \otimes\left[\begin{array}{c}
I_{r-1} \\
0_{r-1}^{\prime}
\end{array}\right] \text {; the } r^{2} \times(r-1) \text { matrix, }
$$

and $X_{12}$ is the $r^{2} \times(r+1)$ matrix, determined from (2), $I_{r-1}$ is the $(r-1) \times(r-1)$ identity matrix, $O_{s t}$ is the $s \times t$ zero matrix, $0_{s}$ is the $s \times 1$ zero vector, and $\otimes$ denotes the Kronecker product. Note that the model matrix $X$ is full column rank, which is $3 r$. In a manner similar to Haber (1985) and Lang and Agresti (1994), the linear space spanned by the columns of the 


\section{ORTHOGONAL DECOMPOSITION FOR QUASI UNIFORM ASSOCIATION MODEL}

matrix $X$ is denoted by $S(X)$ with the dimension $3 r$. Let $U$ be an $r^{2} \times d_{1}$ full column rank matrix, where $d_{1}=r^{2}-3 r=r(r-3)$, such that the linear space spanned by the columns of $U$, that is $S(U)$, is the orthogonal complement of the space $S(X)$. Thus, $U^{\prime} X=O_{d_{1}, 3 r}$. Therefore the QU model is expressed as

$$
h_{1}(p)=0_{d_{1}}
$$

where

$$
h_{1}(p)=U^{\prime} \log p
$$

The MH model may be expressed as

$$
h_{2}(p)=0_{d_{2}} \text {, }
$$

where

$$
d_{2}=r-1, h_{2}(p)=W p,
$$

and $W$ is the $d_{2} \times r^{2}$ matrix with

$$
W=\left[I_{r-1} \otimes 1_{r}^{\prime}, O_{r-1, r}\right]-1_{r}^{\prime} \otimes\left[I_{r-1}, 0_{r-1}\right] .
$$

Therefore, $W^{\prime}=X_{1}-X_{2}$ and thus the column vectors of $W^{\prime}$ belong to the space $S(X)$, that is, $S\left(W^{\prime}\right) \subset S(X)$, hence, $W U=O_{d_{2} d_{1}}$. From Theorem 2, the SQU model may be expressed as

$$
h_{3}(p)=0_{d_{3}},
$$

where

$$
d_{3}=d_{1}+d_{2}=r^{2}-2 r-1,
$$

and

$$
h_{3}(p)=\left(h_{1}^{\prime}, h_{2}^{\prime}\right)^{\prime}
$$

Note that $h_{s}(p), s=1,2,3$ are the vectors of order $d_{s} \times 1$, and $d_{s}, s=1,2,3$, are the numbers of df for testing goodness-of-fit of the QU, MH and SQU models, respectively.
Let $H_{s}(p) \quad s=1,2,3$ denote the $d_{s} \times r^{2}$ matrix of partial derivatives of $h_{s}(p)$ with respect to $p$, that is,

$$
H_{s}(p)=\partial h_{s}(p) / \partial p^{\prime}
$$

Let $\Sigma(p)=\operatorname{diag}(p)-p p^{\prime}$, where $\operatorname{diag}(p)$ denotes a diagonal matrix with $i^{\text {th }}$ component of $p$ as $i^{\text {th }}$ diagonal component. It is observed that

$$
\begin{gathered}
H_{1}(p) p=U^{\prime} 1_{r^{2}}=0_{d_{1}}, \\
H_{1}(p) \operatorname{diag}(p)=U^{\prime}, H_{2}(p)=W .
\end{gathered}
$$

Therefore,

$$
H_{1}(p) \Sigma(p) H_{2}(p)^{\prime}=U^{\prime} W^{\prime}=O_{d_{1} d_{2}},
$$

and $\Delta_{3}=\Delta_{1}+\Delta_{2}$, is obtained where

$$
\Delta_{s}=h_{s}(p)^{\prime}\left[H_{s}(p) \Sigma(p) H_{s}(p)^{\prime}\right]^{-1} h_{s}(p) .
$$

From the asymptotic equivalence of the Wald statistic and the likelihood ratio statistic (Rao, 1973, Sec. 6e.3; Darroch \& Silvey, 1963; Aitchison, 1962), and from (3), (1) is obtained, thus the proof is completed.

\section{Corollary 2} holds:

$$
\text { The following asymptotic equivalence }
$$

$$
G^{2}(S Q I) \simeq G^{2}(Q I)+G^{2}(M H) .
$$

The number of $\mathrm{df}$ for the SQI model equals the sum of numbers of $\mathrm{df}$ for the QI and $\mathrm{MH}$ models.

\section{Example 1}

\section{Results}

Table 1 contains data from a casecontrol study investigating a possible relationship between cataracts and the use of head coverings during the summer. Each case reporting to a clinic for cataract care was 
matched with a control of the same gender and similar age not having a cataract. The row and column categories refer to the frequency with which the subject used head coverings.

The SQU model applied to these data has $G^{2}(S Q U)=10.95$ with $7 \mathrm{df}$. Thus, the SQU model fits these data well. Under this model, the maximum likelihood estimate of $\theta$ is $\hat{\theta}=0.808$. The SQU model indicates the structure of both the $\mathrm{S}$ and QU models. Therefore, under the SQU model, the probability that using a head covering for one case in a pair is always or almost always, and for the control in the pair is never, is estimated to equal the probability of using a head covering for a case in the pair is never, and for control in the pair is always or almost always.
For local $2 \times 2$ tables that do not contain a cell on the main diagonal, the odds that using head covering for a case in a pair is $s+1$ instead of $s$ is estimated to be $\hat{\theta}=0.808$ times when that for the control in the pair is $t+1$ than when it is $t$. For $i<j$ and $s<t$ with $i \neq s$, $i \neq t, j \neq s, j \neq t$, the odds that the using head covering case in a pair is $j$ instead of $i$ is estimated to be $(0.808)^{(j-i)(t-s)}$ times higher when that for the control in the pair is $t$ than when it is $s$. For example, the odds that the using a head covering for a case in a pair is never instead of frequency is estimated to be $0.426\left[=(0.808)^{4}\right]$ times higher when that for control in the pair is occasionally than when it is always or almost always.

Table 1: Case-Control Study Investigating a Possible Relationship between Cataracts and the Use of Head Coverings during the Summer

\begin{tabular}{|c|c|c|c|c|}
\hline \multirow{2}{*}{ Cataract Case } & \multicolumn{4}{|c|}{ Control } \\
\cline { 2 - 5 } & $\begin{array}{c}\text { Always or } \\
\text { Almost Always } \\
(1)\end{array}$ & $\begin{array}{c}\text { Frequently } \\
(2)\end{array}$ & $\begin{array}{c}\text { Occasionally } \\
(3)\end{array}$ & $\begin{array}{c}\text { Never } \\
(4)\end{array}$ \\
\hline \multirow{2}{*}{ Always or Almost Always (1) } & 29 & 3 & 3 & 4 \\
& $(29.00)^{*}$ & $(4.22)$ & $(5.12)$ & $(6.16)$ \\
\hline Frequently (2) & 5 & 0 & 1 & 1 \\
\hline Occasionally (3) & $(4.22)$ & $(0.00)$ & $(1.16)$ & $(1.12)$ \\
\hline Never (4) & $(5.12)$ & $(1.16)$ & $(2.00)$ & $(0.72)$ \\
\hline & $(6.16)$ & $(1.12)$ & $(0.72)$ & $(0.00)$ \\
\hline
\end{tabular}

*Note: The parenthesized values are the maximum likelihood estimates of expected frequencies under the SQU model. 


\section{ORTHOGONAL DECOMPOSITION FOR QUASI UNIFORM ASSOCIATION MODEL}

Example 2

Table 2 contains data from the Los Angeles study of endometrial cancer. These data were obtained from 59 matched pairs using four dose levels of conjugated oestrogen: (1) none, (2) $0.1-0.299 \mathrm{mg}$, (3) $0.3-0.625 \mathrm{mg}$, and (4) $0.626+\mathrm{mg}$.

Table 3 shows that the SQU and $\mathrm{MH}$ models fit the data poorly while the QU model fits the data well. From Theorem 2, the poor fit of the SQU model may be said to be caused by the influence of the lack of structure of the $\mathrm{MH}$ model rather than the QU model. Because the QU model fits the data in Table 2 well, under this model, the cell probabilities $\left\{p_{i j}\right\}$ have a uniform local association for cells off the main diagonal of the table.

Table 2: Average Doses of Conjugated Oestrogen Used By Cases and Matched Controls:

Los Angeles Endometrial Cancer Study

(Breslow \& Day, 1980, p. 185)

\begin{tabular}{|c|c|c|c|c|c|}
\hline \multirow{2}{*}{$\begin{array}{c}\text { Average Dose } \\
\text { for Case } \\
(\mathrm{mg} / \text { day })\end{array}$} & \multicolumn{4}{|c|}{ Average Dose for Control (mg/day) } & \multirow{2}{*}{ Total } \\
\cline { 2 - 6 } & 0 & $0.1-0.299$ & $\begin{array}{c}0.3-0.625 \\
(1)\end{array}$ & $\begin{array}{c}0.625+ \\
(4)\end{array}$ & \\
\hline $0(1)$ & 6 & 2 & 3 & 1 & 12 \\
\hline $0.1-0.299(2)$ & 9 & 4 & 2 & 1 & 16 \\
\hline $0.3-0.625(3)$ & 9 & 2 & 3 & 1 & 15 \\
\hline $0.625+(4)$ & 12 & 1 & 2 & 1 & 16 \\
\hline Total & 36 & 9 & 10 & 4 & 59 \\
\hline
\end{tabular}

Table 3: Likelihood Ratio Chi-squared Values $G^{2}$ for Models Applied to Tables 1 and 2

\begin{tabular}{|c|c|c|c|c|}
\hline \multirow{2}{*}{ Applied Models } & \multicolumn{2}{|c|}{ Table1 } & \multicolumn{2}{c|}{ Table2 } \\
\cline { 2 - 5 } & $\begin{array}{c}\text { Degrees of } \\
\text { Freedom }\end{array}$ & $G^{2}$ & $\begin{array}{c}\text { Degrees of } \\
\text { Freedom }\end{array}$ & $G^{2}$ \\
\hline QI & 5 & 6.99 & 5 & 0.77 \\
\hline QU & 4 & 6.52 & 4 & 0.69 \\
\hline SQI & 8 & 11.56 & 8 & $19.98^{*}$ \\
\hline SQU & 7 & 10.95 & 7 & $19.86^{*}$ \\
\hline S & 6 & 8.29 & 6 & $19.27^{*}$ \\
\hline QS & 3 & 3.85 & 3 & 0.46 \\
\hline MH & 3 & 4.38 & 3 & $19.12^{*}$ \\
\hline
\end{tabular}

* means are significant at the 0.05 level 


\section{Conclusion}

This article gives the decomposition of the SQU model and shows the orthogonality of test statistics. As observed in the examples, Theorem 2 would be useful for explaining the reason for the poor fit when the SQU model fits the data poorly.

From Theorem 3 it may be noted that the likelihood ratio statistic for testing goodnessof-fit of the SQU model - assuming that the QU model holds true - is $G^{2}(S Q U)-G^{2}(Q U)$ and this is asymptotically equivalent to the likelihood ratio statistic for testing goodness-offit of the $\mathrm{MH}$ model, that is, $G^{2}(M H)$. Namely, $G^{2}(S Q U)-G^{2}(Q U)$ would be used for testing goodness-of-fit of the MH model.

Suppose that model $\Omega_{3}$ holds if and only if both models $\Omega_{1}$ and $\Omega_{2}$ hold, where the number of df for $\Omega_{3}$ equals the sum of numbers of $\mathrm{df}$ for $\Omega_{1}$ and $\Omega_{2}$. Darroch and Silvey (1963) described that (i) when the asymptotic equivalence, then

$$
G^{2}\left(\Omega_{3}\right) \simeq G^{2}\left(\Omega_{1}\right)+G^{2}\left(\Omega_{2}\right)
$$

holds, if both $\Omega_{1}$ and $\Omega_{2}$ are accepted (at the $\alpha$ significance level) with high probability, then $\Omega_{3}$ would be accepted; however, (ii) when equation (4) does not hold, it is possible for an incompatible situation to arise where both $\Omega_{1}$ and $\Omega_{2}$ are accepted with high probability but $\Omega_{3}$ is rejected with high probability (Darroch and Silvey (1963) show an interesting example). For the orthogonal decomposition of the SQU model into the QU and MH models, such an incompatible situation would not arise in terms of Theorem 3. Therefore, the orthogonal decomposition of the SQU model obtained herein is useful for analyzing data.
References

Agresti, A. (1996). An introduction to categorical data analysis. New York: Wiley.

Aitchison, J. (1962). Large-sample restricted parametric tests. Journal of the Royal Statistical Society, Ser. B, 24, 234-250.

Bishop, Y. M. M., Fienberg, S. E., \& Holland, P. W. (1975). Discrete multivariate analysis: theory and practice. Cambridge, MA: The MIT Press.

Bowker, A. H. (1948). A test for symmetry in contingency tables. Journal of the American Statistical Association, 43, 572-574.

Breslow, N. E., \& Day, N. E. (1980). Statistical methods in cancer research, Vol. I: The analysis of case-control studies. Lyon, France: International Agency for Research on Cancer.

Caussinus, H. (1965). Contribution à l'analyse statistique des tableaux de corré lation. Annales de la Faculté des Sciences de l'Université de Toulouse, 29, 77-182.

Darroch, J. N., \& Ratcliff, D. (1972). Generalized iterative scaling for log-linear models. Annals of Mathematical Statistics, 43, 1470-1480.

Darroch, J. N., \& Silvey, S. D. (1963). On testing more than one hypothesis. Annals of Mathematical Statistics, 34, 555-567.

Goodman, L. A. (1979). Simple models for the analysis of association in crossclassifications having ordered categories. Journal of the American Statistical Association, 74, 537-552.

Goodman, L. A. (1985). The analysis of cross-classified data having ordered and/or unordered categories: association models, correlation models, and asymmetry models for contingency tables with or without missing entries. Annals of Statistics, 13, 10-69.

Haber, M. (1985). Maximum likelihood methods for linear and log-linear models in categorical data. Computational Statistics and Data Analysis, 3, 1-10.

Lang, J. B. (1996). On the partitioning of goodness-of-fit statistics for multivariate categorical response models. Journal of the American Statistical Association, 91, 10171023. 


\section{ORTHOGONAL DECOMPOSITION FOR QUASI UNIFORM ASSOCIATION MODEL}

Lang, J. B., \& Agresti, A. (1994). Simultaneously modeling joint and marginal distributions of multivariate categorical responses. Journal of the American Statistical Association, 89, 625-632.

Rao, C. R. (1973). Linear statistical inference and its applications $\left(2^{\text {nd }} E d\right.$.). New York: Wiley.

Read, C. B. (1977). Partitioning chisquare in contingency tables: a teaching approach. Communications in Statistics-Theory and Methods, 6, 553-562.

Stuart, A. (1955). A test for homogeneity of the marginal distributions in a two-way classification. Biometrika, 42, 412-416.
Tahata, K., \& Tomizawa, S. (2008). Orthogonal decomposition of point-symmetry for multiway tables. Advances in Statistical Analysis, 92, 255-269.

Tomizawa, S., \& Tahata, K. (2007). The analysis of symmetry and asymmetry: orthogonality of decomposition of symmetry into quasi-symmetry and marginal symmetry for multi-way tables. Journal de la Société Francaise de Statistique, 148, 3-36. 